\title{
Lattice guiding for sputter deposition of single domain (Sr0.6Ba0.4)Nb206 ferroelectric thin films
}

Bhaskaran, Madhu; Sriram, Sharath; Rodriguez, Brian; Devendra, Geethaka; Latham, Kay; McCulloch, Dougal; Mitchell, Arnan

https://researchrepository.rmit.edu.au/esploro/outputs/9921858308101341/filesAndLinks?institution=61RMIT_INST\&index=null

Bhaskaran, M., Sriram, S., Rodriguez, B., Devendra, G., Latham, K., McCulloch, D., \& Mitchell, A. (2012). Lattice guiding for sputter deposition of single domain (Sr0.6Ba0.4)Nb2O6 ferroelectric thin films. CrystEngComm, 14(2), 359-361. https://doi.org/10.1039/c1ce06112g

Document Version: Accepted Manuscript

Published Version: https://doi.org/10.1039/c1ce06112g

Repository homepage: https://researchrepository.rmit.edu.au

(C) The Royal Society of Chemistry 2012

Downloaded On 2023/04/27 00:27:36 +1000 
Thank you for downloading this document from the RMIT Research Repository.

The RMIT Research Repository is an open access database showcasing the research outputs of RMIT University researchers.

RMIT Research Repository: http://researchbank.rmit.edu.au/

\section{Citation:}

Bhaskaran, M, Sriram, S, Rodriguez, B, Devendra, G, Latham, K, McCulloch, D and Mitchell, A 2011, 'Lattice guiding for sputter deposition of single domain (Sr0.6Ba0.4)Nb2O6 ferroelectric thin films', CrystEngComm, vol. 14, no. 2, pp. 359-361.

See this record in the RMIT Research Repository at:

http://researchbank.rmit.edu.au/view/rmit:14143

Version: Accepted Manuscript

Copyright Statement: (C) The Royal Society of Chemistry 2012

Link to Published Version:

http://dx.doi.org/10.1039/c1ce06112g 


\title{
Lattice guiding for sputter deposition of single domain $\left(\mathrm{Sr}_{0.6} \mathrm{Ba}_{0.4}\right) \mathrm{Nb}_{2} \mathrm{O}_{6}$ ferroelectric thin films $\dagger$
}

\author{
Madhu Bhaskaran, ${ }^{* a}$ Sharath Sriram, ${ }^{* a}$ Brian J. Rodriguez, ${ }^{b}$ Geethaka C. Devendra, ${ }^{a}$ Kay Latham, ${ }^{c}$ \\ Dougal G. McCulloch ${ }^{c}$ and Arnan Mitchell ${ }^{a, d}$ \\ 5 Received (in XXX, XXX) Xth XXXXXXXXX 2011, Accepted Xth XXXXXXXXX 2011 \\ DOI: 10.1039/c0xx00000x
}

Lattice guiding is used to synthesize preferentially oriented thin films of $\left(\mathrm{Sr}_{0.6} \mathrm{Ba}_{0.4}\right) \mathrm{Nb}_{2} \mathrm{O}_{6}$ by $\mathrm{RF}$ magnetron sputtering, with the film-substrate crystallographic relationship 10 determined. These ferroelectric thin films guide light at $\mathbf{5 3 2}$ and $633 \mathrm{~nm}$ and have a single domain structure that can be written with permanent, sub-micron, anti-parallel domains.

Ferroelectric crystals are capable of manipulating light based on the distribution of their polar domains enabling functionalities 15 such as frequency conversion and quantum information processing. ${ }^{1}$ The distribution of the ferroelectric domains in the crystals determine the conversion characteristics and efficiency, with random distribution having broadband performance and periodic distribution enabling highly coherent conversion. ${ }^{1}$ To 20 enhance the performance of ferroelectric materials for applications in frequency conversion and quantum information processing, confined light propagation in thin film waveguides is advantageous. ${ }^{2}$ This requires the growth of near epitaxial and domain patternable ferroelectric thin films, the combination of 25 which allows waveguiding and efficient light manipulation.

Experimental demonstrations have shown that strontium barium niobate $(\mathrm{SBN})$ ferroelectric crystals with random ferroelectric domains are effective second harmonic generation (SHG) materials, with a particular SBN composition of $30\left(\mathrm{Sr}_{0.6} \mathrm{Ba}_{0.4}\right) \mathrm{Nb}_{2} \mathrm{O}_{6}$ (denoted henceforth as $\mathrm{SBN} 60$, due to $60 \% A$ site strontium) proving to be very efficient. ${ }^{1}$ Synthesis of SBN60 in thin film form will enable controlled patterning of ferroelectric domains, allowing quasi phase matching for forward and backward SHG, in addition to facilitating incorporation of 35 waveguides and couplers, creating a platform for hybrid integration.

To achieve growth of epitaxial thin films, pulsed laser deposition (PLD) is the technique usually adopted., ${ }^{2,3}$ PLD has also been used to achieve epitaxial thin films of SBN on $\mathrm{MgO}$ 40 substrates. ${ }^{2,4}$ The use of PLD in this instance is encumbered by two major drawbacks. Firstly, the laser ablation process results in large crystalline deposits on the surface on the thin films. ${ }^{3}$ While this can be reduced by off-axis depositions, it cannot be fully controlled. Secondly, the distribution of the plasma plume limits 45 the growth of uniform epitaxial films by PLD to relatively small areas. Considering potential applications of epitaxial SBN thin films for SHG in integrated optic devices, uniform and high quality films over large areas to enable devices $10-50 \mathrm{~mm}$ long is critical for maximum light interaction with the materials and 50 efficient conversion in the case of SHG. ${ }^{\mathbf{1 , 2}}$ Moreover, even with epitaxial PLD films, single domain SBN60 films which can be controllably patterned have not been demonstrated.

In this work, we utilise RF magnetron sputtering, which is reputed for large area and uniform thin film quality ${ }^{3}$ to deposit ${ }_{55} \mathrm{SBN}$ thin films. RF magnetron sputtering is known to generally result in polycrystalline films, unless optimised conditions or lattice guiding or a combination of the two are employed. ${ }^{3,5}$ The sputtering process was carried out using a stoichiometric $\left(\mathrm{Sr}_{0.6} \mathrm{Ba}_{0.4}\right) \mathrm{Nb}_{2} \mathrm{O}_{6}$ target at $200 \mathrm{~W}$ forward power in an argon 60 atmosphere (see ESI $\dagger$ for details). SBN60 has a tetragonal structure with lattice parameters $a$ and $c$ being 12.46 and $3.93 \AA$, respectively. ${ }^{6}$ In order to synthesise highly oriented thin films, a combination of lattice guiding and thermodynamics was utilised. Strontium titanate $\left(\mathrm{SrTiO}_{3}, \mathrm{STO}\right)$ and $0.5 \mathrm{wt} \%$ niobium-doped ${ }_{65}$ strontium titanate $(\mathrm{Nb}: \mathrm{STO})$ substrates of $(001)$ orientation with lattice parameter of $3.91 \AA$ were chosen to minimise misfit strain. ${ }^{7}$ This level of niobium doping does not influence the substrate crystallography, but provides DC conductivity utilised for ferroelectric characterisation. The deposition was carried out 70 at a substrate temperature of $700{ }^{\circ} \mathrm{C}$, followed by controlled cooling at $5{ }^{\circ} \mathrm{C} \mathrm{min}^{-1}$ to maximise the crystalline quality of the films. ${ }^{8}$ The resulting thin film deposition rate was $\sim 175 \mathrm{~nm} \mathrm{~h}^{-1}$.

X-ray photoelectron spectroscopy (XPS) was utilised to determine the composition of the deposited thin films. The 75 experimental conditions and results are described in detailed in the ESI $\uparrow$ (see Fig. S1). The composition of the films was verified as matching that of the sputtering target and the desired SBN60 composition. The crystallography of the thin films was determined using X-ray diffraction (XRD) (see ESI $\uparrow$ for ${ }_{80}$ experimental details). The resulting diffractogram is shown in Fig. 1 and was indexed based on reference powder diffraction files 01-072-6171 for SBN61 ${ }^{6}$ and 00-035-0734 for $\mathrm{STO}^{7}$ from the International Centre for Diffraction Data (ICDD). The overlap of the (002) planes of SBN60 and STO observed in Fig. 1 is ${ }_{85}$ indicative of the guiding effect exerted by STO on the orientation of the SBN60 thin films. The diffractogram also indicates that the SBN thin films are preferentially oriented given the dominant (001) and (002) peaks. Identical XRD results were obtained for SBN60 on Nb:STO substrates. 


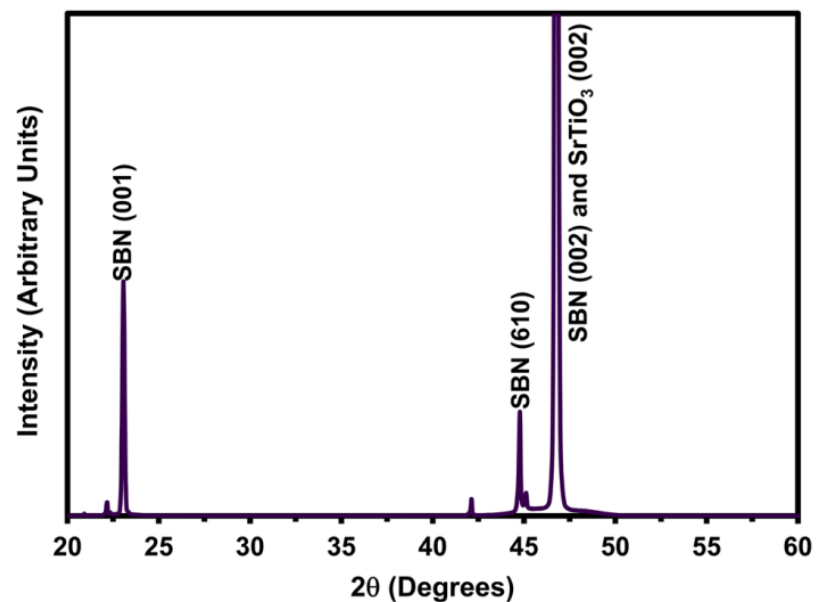

Fig. 1 X-ray diffraction of SBN60 thin film on STO substrate showing strong preferential orientation.

Cross-sectional transmission electron microscopy (XTEM) 5 was used to determine the microstructure, thickness, and crystallography of the thin films. Figure 2 presents the high resolution cross-sectional microstructure of the SBN60 thin films on the STO substrate, which shows a polycrystalline microstructure and a film thickness of $170-178 \mathrm{~nm}$ for a $1 \mathrm{~h}$

10 deposition. Selected area electron diffraction (SAED) was used to determine the crystallographic relationship between the crystallites in the SBN60 thin film and the STO substrate. The SAED pattern obtained in the vicinity of the film-substrate interface is shown in Fig. 3(a) (and Fig. S2 in the ESI $\dagger$ ). The 15 diffraction pattern shows contributions from both the STO (red) and SBN60 (green). Figures 3(b) and 3(c) show each of these diffraction patterns indexed and reveal that the electron beam is [011] direction in STO and [001] in SBN60. The diffraction pattern show that there is a strong crystallographic orientation 20 relationship between $\mathrm{SrTiO}_{3}$ and $\mathrm{SBN} 60$ with the overlap of the (211) $\mathrm{STO}$ and (200) $\mathrm{SrTiO}_{3}$ diffraction spots. The $\sim 35^{\circ}$ tilt angle between the diffraction patterns corresponds to the $(211)^{\wedge}(200)$ interplanar angle. It can also be seen in Fig. 3(c) (and Fig. S2 in the ESI $\uparrow$ ) that there are intermediate spots between the indexed 25 spots. These arise due to SBN60 having a superlattice structure of strontium niobate and barium niobate. ${ }^{9}$

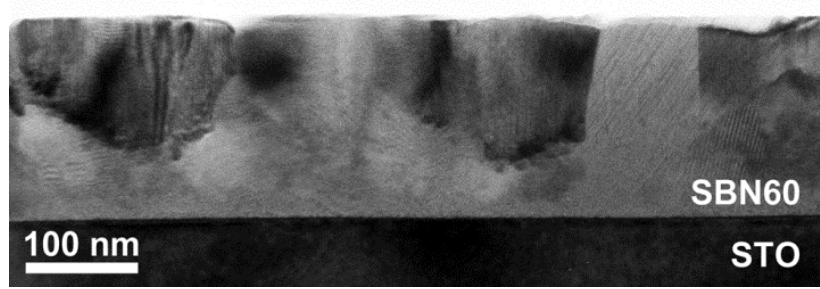

Fig. 2 Cross-sectional transmission electron micrograph of the SBN60 thin film on a STO substrate showing a polycrystalline microstructure.

30 Having established lattice guided growth of preferentially oriented thin films of SBN60 on STO substrates, the ability of these films to serve as light guiding layers and domain patternable ferroelectrics was examined by prism coupling and piezoresponse force microscopy, respectively.

Prism coupling is an efficient technique for coupling light into a thin film, using a precisely polished prism, overcoming the need to fabricate a waveguide. ${ }^{10}$ The observation of coupling modes is indicative of the ability of the thin film layer to guide light in a film-substrate arrangement, and sufficient number of modes can 40 be utilised to extract the refractive index and thickness of the thin film. A Metricon prism coupler was used to study the SBN60 thin films (see ESI $\dagger$ for details). The measurements were undertaken at 532 and $633 \mathrm{~nm}$ to determine the ability of the films to guide both wavelengths. In order to have sufficient film thickness to 45 support optical modes, the films utilised for these experiments were obtained by a $6 \mathrm{~h}$ deposition $(\sim 1.05 \mu \mathrm{m})$.

Figure 4 presents prism coupling results for the SBN60-STO film-substrate structure. Dips seen in the spectra correspond to optical modes supported by the film with the position of each 50 mode representing the effective modal index $\beta$. Optical modes are supported by the SBN60 thin film at both 532 and $633 \mathrm{~nm}$, demonstrating the ability of these films to guide light. From the effective modal indices $\beta$, the ordinary refractive index $\left(n_{\mathrm{o}}\right.$, TE mode) was determined at 532 and $633 \mathrm{~nm}$ as 2.367 and 2.334 , 55 respectively. This refractive index is in agreement with 2.30 at $633 \mathrm{~nm}$ obtained for highly oriented SBN60 thin films on platinised $\mathrm{MgO}$ substrates. ${ }^{11}$ The film thickness extracted from the prism coupling measurements was $1.02 \mu \mathrm{m}$, which matches the expected value of $\sim 1.05 \mu \mathrm{m}$.
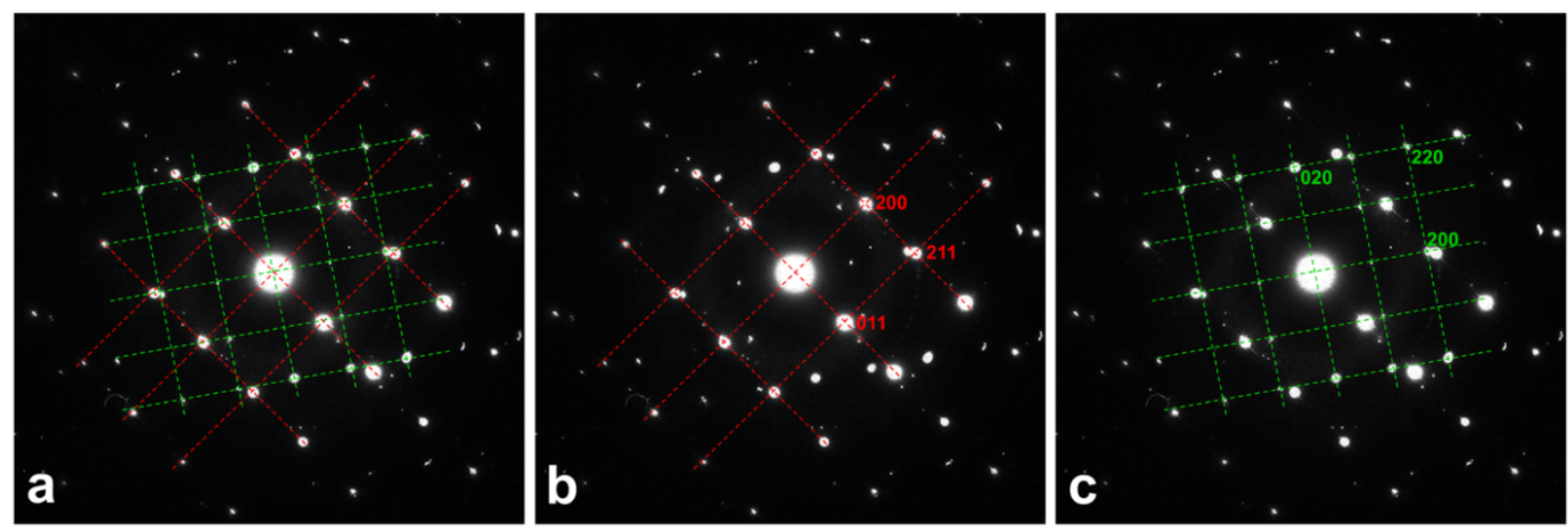

Fig. 3 (a) Selected area diffraction pattern (SAED) taken in the vicinity of the STO/SBN60 interface. (b) Indexed SAED for STO. (c) Indexed SAED for the SBN60 thin film. The pattern for STO (red) is tilted at $\sim 35^{\circ}$ to SBN60 (green), the angle between (211) STO and (002) SBN60. 


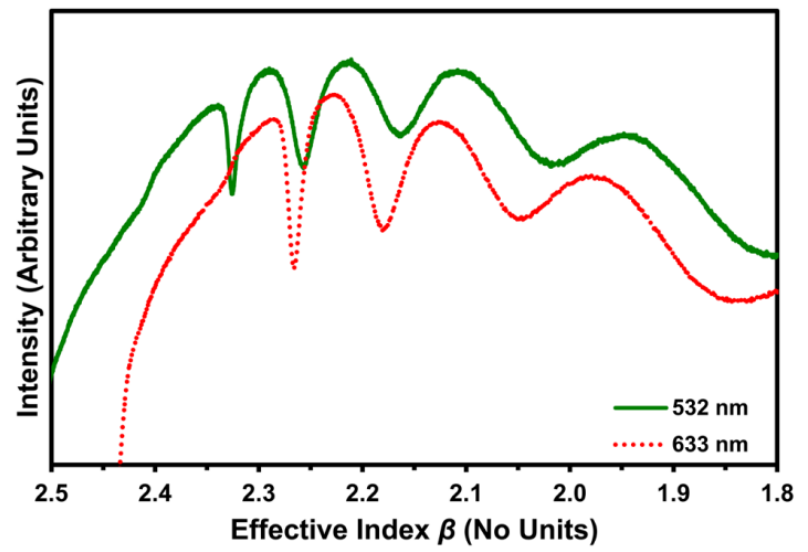

Fig. 4 Prism coupling spectra for SBN60 thin films. The modes observed confirm optical guiding at 532 and $633 \mathrm{~nm}$.

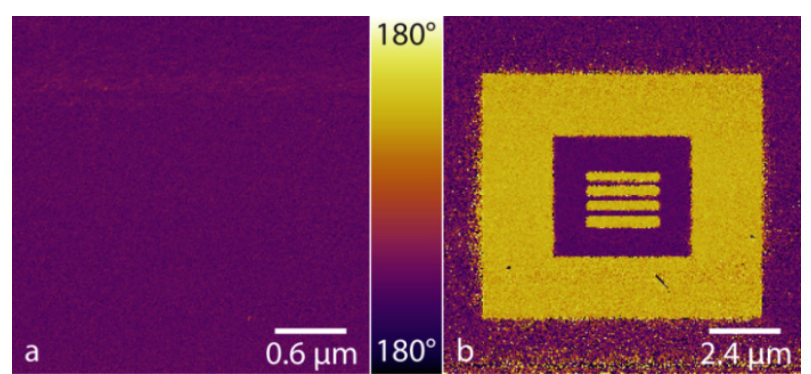

5 Fig. 5 Piezoresponse force microscopy results showing phase maps of ferroelectric domains in SBN60 thin films on Nb:STO. The single domain characteristic of the films is shown in (a). The ability to write micro- and nano-scale domains with a $\pm 6 \mathrm{~V}$ tip bias is shown in (b), where the contrast depicts anti-parallel $180^{\circ}$ domains.

10 Piezoresponse force microscopy (PFM) is increasingly used to characterise the ferroelectric properties of thin films, and, in particular, to study the distribution and polarity of domains. ${ }^{12,13}$ The technique uses an atomic force microscope (AFM) to determine piezoelectric displacements under applied bias. ${ }^{\mathbf{1 2 , 1 3}}$ The

${ }_{15}$ SBN60 thin films on STO and Nb:STO substrates were both studied by PFM, with $\mathrm{Nb}$ :STO providing a conductive substrate to serve as the bottom ground electrode, while in the case of STO the piezoelectric response is purely determined by bias-induced electric field at the AFM tip. The surface topography of these 20 films revealed a very smooth surface (see ESI†, Fig. S3), with root mean square roughness of $126 \mathrm{pm}(\sim 0.1 \mathrm{~nm})$. PFM phase maps, which provide information about the orientation of the ferroelectric polarization, for a set of measurements are presented in Fig. 5. The as-deposited thin films have a uniform piezoelectric 25 phase response as seen in Fig. 5(a) and hence a uniform polarization direction, and thus appear to be comprised exclusively of a single ferroelectric domain. Using a $\pm 6 \mathrm{~V}$ bias applied to the AFM tip, inverse domains could be written in the film with sub-micron resolution, as shown in Fig. 5(b). Some 30 topography changes were observed, which did not affect the switchability of the domains. No in-plane displacements were observed, confirming the domains being formed were antiparallel $180^{\circ}$ ferroelectric domains, which are usually observed for tetragonal unit cell structure materials, as in the case of ${ }_{35}$ SBN60. ${ }^{13,14}$ Written domains remained stable for at least one week.

In summary, we have demonstrated the use of lattice guiding for the synthesis of preferentially oriented thin films of SBN60 on STO substrates. The interfacial crystallographic relationship was 40 examined using XRD and SAED. These films are shown to be suitable for optical waveguiding at 532 and $633 \mathrm{~nm}$. The asdeposited single domain ferroelectric structure of the films can be patterned with sub-micron inverse domains. These SBN60 thin films have the potential to be an efficient platform for high 45 efficiency nonlinear optical processing.

\section{Acknowledgements}

This work was supported by the Australian Research Council (ARC) through infrastructure funding (LE0882246 and LE0989615), Discovery Project DP1092717 (MB), and the ${ }_{50}$ Centres of Excellence program (AM). MB and SS acknowledge ARC Australian Post-Doctoral Fellowships through DP1092717 and DP110100262, respectively. MB and BJR acknowledge support from the RMIT Foundation. The authors thank Johan du Plessis for assistance with X-ray photoelectron 55 spectroscopy.

\section{Notes and references}

a Microplatforms Research Group, School of Electrical and Computer Engineering, RMIT University, GPO Box 2476, Melbourne, Victoria 3001, Australia. E-mail: madhu.bhaskaran@gmail.com, 60 sharath.sriram@gmail.com

${ }^{b}$ Conway Institute of Biomolecular and Biomedical Research, University College Dublin, Belfield, Dublin 4, Ireland

School of Applied Sciences, RMIT University, GPO Box 2476, Melbourne, Victoria 3001, Australia

${ }_{65}{ }^{d}$ ARC Centre for Ultrahigh bandwidth Devices for Optical Systems, RMIT University, GPO Box 2476, Melbourne, Victoria 3001, Australia

$\dagger$ Electronic Supplementary Information (ESI) available: Experimental conditions for thin film deposition and for characterisation processes; Xray photoelectron spectroscopy results; as-collected electron diffraction 70 pattern; topography of film surface. See DOI: $10.1039 / \mathrm{c} 0 \mathrm{xx} 00000 \mathrm{x} /$

1 J. Trull et al., Opt. Express, 2007, 15, 15868; V. Roppo et al., Opt. Express, 2010, 18, 4012.

2 S. Schwyn Thöny, K. E. Youden, J. S. Harris, Jr. and L. Hesselink, Appl. Phys. Lett., 1994, 65, 2018.

753 K. Wasa, M. Kitabatake and H. Adachi, Thin Film Materials Technology: Sputtering of Compound Materials, Springer-Verlag GmbH \& Co. KG, Heidelberg, Germany, 2004.

4 D. Trivedi, P. Tayebati and M. Tabat, Appl. Phys. Lett., 1996, 68, 3227.

805 S. Sriram, M. Bhaskaran, D. R. G. Mitchell and A. Mitchell, Cryst. Growth Des., 2010, 10, 761.

6 Powder Diffraction Pattern Files, International Centre for Diffraction Data (ICDD), Newtown Square, PA 19073, Card 01-072-6171.

7 Powder Diffraction Pattern Files, International Centre for Diffraction 85 Data (ICDD), Newtown Square, PA 19073, Card 00-035-0734.

8 S. Sriram, M. Bhaskaran and A. S. Holland, Semicond. Sci. Technol., 2006, 21, 1236

9 L. A. Bursill and P. J. Lin, Phil. Mag. B, 1986, 54, 157; L. A. Bursill and P. J. Lin, Acta Crystallogr. B, 1987, 43, 49.

9010 P. K. Tien, R. Ulrich and R. L. Martin, Appl. Phys. Lett., 1969, 14, 291; P. K. Tien and R. Ulrich, J. Opt. Soc. Am., 1970, 60, 1325; R. Ulrich, J. Opt. Soc. Am., 1970, 60, 1337.

11 M. Cuniot-Ponsard, J. M. Desvignes, A. Bellemain and F. Bridou, $J$. Appl. Phys., 2011, 109, 014107

9512 B. J. Rodriguez, C. Callahan, S. V. Kalinin and R. Proksch, Nanotechnol., 2007, 18, 475504.

13 S. V. Kalinin, A. N. Morozovska, L. Q. Chen and B. J. Rodriguez, Rep. Prog. Phys., 2010, 73, 056502.

14 D. Damjanovic, Rep. Prog. Phys., 1998, 61, 1267; J. Guyonnet et al., $100 \quad$ Appl. Phys. Lett., 2009, 95, 132902. 